\title{
Effects of Medicinal Plant Extracts and Photosensitization on Aflatoxin Producing Aspergillus flavus (Raper and Fennell)
}

\author{
Loise M. Njoki, Sheila A. Okoth, and Peter M. Wachira \\ School of Biological Sciences, University of Nairobi, P.O. Box 30197-00100, Nairobi, Kenya \\ Correspondence should be addressed to Loise M. Njoki; infoloisemumbi2030@gmail.com
}

Received 12 January 2017; Revised 29 March 2017; Accepted 13 April 2017; Published 2 May 2017

Academic Editor: Todd R. Callaway

Copyright (C) 2017 Loise M. Njoki et al. This is an open access article distributed under the Creative Commons Attribution License, which permits unrestricted use, distribution, and reproduction in any medium, provided the original work is properly cited.

\begin{abstract}
This study was undertaken with an aim of exploring the effectiveness of medicinal plant extracts in the control of aflatoxin production. Antifungal properties, photosensitization, and phytochemical composition of aqueous and organic extracts of fruits from Solanum aculeastrum, bark from Syzygium cordatum, and leaves from Prunus africana, Ocimum lamiifolium, Lippia kituiensis, and Spinacia oleracea were tested. Spores from four-day-old cultures of previously identified toxigenic fungi, UONV017 and UONV003, were used. Disc diffusion and broth dilution methods were used to test the antifungal activity. The spores were suspended in $2 \mathrm{ml}$ of each extract separately and treated with visible light $(420 \mathrm{~nm})$ for varying periods. Organic extracts displayed species and concentration dependent antifungal activity. Solanum aculeastrum had the highest zones of inhibition diameters in both strains: UONV017 (mean $=18.50 \pm 0.71 \mathrm{~mm}$ ) and UONV003 (mean $=11.92 \pm 0.94 \mathrm{~mm})$ at $600 \mathrm{mg} / \mathrm{ml}$. Aqueous extracts had no antifungal activity because all diameters were below $8 \mathrm{~mm}$. Solanum aculeastrum had the lowest minimum inhibitory concentration at $25 \mathrm{mg} / \mathrm{ml}$ against $A$. flavus UONV017. All the plant extracts in combination with light reduced the viability of fungal conidia compared with the controls without light, without extracts, and without both extracts and light. Six bioactive compounds were analyzed in the plant extracts. Medicinal plant extracts in this study can control conidia viability and hence with further development can control toxigenic fungal spread.
\end{abstract}

\section{Introduction}

Aspergillus flavus is ubiquitous, saprophytic, and a weak parasite [1]. The fungus contaminates a wide range of cereals and nuts like maize, wheat, sorghum, and groundnuts, which serve as staple foods in most parts of Africa. Toxigenic $A$. flavus have been reported to contaminate these products and produce aflatoxins which are carcinogenic, mutagenic, and lethal fungal metabolites [2-4]. Aflatoxins have been classified as class 1 poisons by the International Agency for Research on Cancer (IARC) [5]. Aflatoxins also contaminate feed; hence products like meat, milk, cheese, and eggs get contaminated when animals consume the aflatoxin contaminated feed $[6,7]$. Aflatoxin $\mathrm{B} 1$ is the major type of aflatoxins produced by A. flavus [3]. Aspergillus flavus is the main fungi producing aflatoxin [1].

Aflatoxicosis was first reported in Kenya in 1982 [8]. More outbreaks have since been recorded in 2001, 2004, 2006, 2008, and $2010[9,10]$. Records of aflatoxin contamination in food and feed are widespread in tropical and subtropical regions where climatic conditions and storage practices favor growth of fungi [11]. Aflatoxin production is influenced by aeration, moisture, temperature, and substrate and the control methods under trials like biological [12], cultural, and chemical ones [13] are all based on manipulation of these factors. Many African countries are now in the process of including regulation framework within their food policies to help control exposure to aflatoxins [1].

Traditionally, many plants have been used successfully for medicinal purposes [4]. Aromatic substances in plants, specifically secondary metabolites like alkaloids, flavonoids, saponins, glycosides, and tannins, are able to protect plants from invaders such as fungi, bacteria, and nematodes [14]. According to World Health Organization (2001), 80\% of African and Asian communities rely on traditional herbal medicines for primary healthcare. This is because herbal medicines are safer and cheaper compared to synthetic medicines $[15,16]$. 
This study exploited the ability of known medicinal plant extracts to control the growth of A. flavus conidia. Photosensitization has also been reported to kill both metabolically active and dormant structures such as conidia, unlike conventional fungicides that kill only metabolically active cells [17]. It involves hitting of a photosensitizer by light of a specific wavelength, which makes the photosensitizer reactive thereby killing the toxigenic cells [18]. The technique has been reported as a safe and a potential control of mycotoxigenic fungi [19]. However, very few photosensitizers have been approved clinically for use against toxigenic microbes, hence needing identification of safe photosensitizers. Plant extracts, on the other hand, are biodegradable and hence environmentally friendly [20]. The aim of this study is to determine the antifungal activity and phytochemical composition of the plants extracts. The ability of visible light to stimulate the bioactive compounds in the plant extracts (photosensitization) and hence increase in the antifungal activity of the extracts against toxigenic $A$. flavus which causes aflatoxin production will also be tested.

\section{Materials and Methods}

2.1. Collection of Plant Material. Five plants known for their medicinal value were collected from Gakoe forest in Gatundu district, Central Region of Kenya. These were Ocimum lamiifolium leaves (LMM 2015/05), Prunus africana leaves (LMM 2015/03), Solanum aculeastrum fruits (LMM 2015/01), Lippia kituiensis leaves (LMM 2015/04), and Syzygium cordatum bark (LMM 2015/02). Fresh leaves of Spinacia oleracea (LMM 2015/06) were also collected from the local market. The identity of the plants was confirmed using reference material from the University of Nairobi herbarium where voucher specimens were deposited.

2.2. Crude Plant Extract Preparation. The selected plant parts were air-dried at room temperature, chopped, and ground into powder. Dichloromethane-methanol (1:1) mixture was used for organic extraction. Two hundred and fifty grams of each ground extract was soaked in $1 \mathrm{~L}$ of the organic solvents for 48 hours. A rotary evaporator was used to filter and concentrate the organic extract, hence obtaining a semisolid residue for use [4]. Distilled water was used for aqueous extraction. Two hundred and fifty grams of each ground extract was soaked in $500 \mathrm{ml}$ of distilled water in a glass beaker sealed with aluminum foil for five days. The extract was then filtered using Whatman number 1 filter paper. The filtrate was evaporated and dried using a freeze-drier to get powder [21]. The resulting products were stored at $4^{\circ} \mathrm{C}$.

2.3. Preparation of Fungal Spore Suspension. Toxigenic A. flavus strains used in this study were obtained from the School of Biological Sciences Mycology Culture Collection. The isolates used were UONV017 and UONV003 and they had been tested for toxigenicity through molecular characterization according to [3]. The isolates were transferred from the stock cultures into sterile PDA plates and incubated for 4 days at $29^{\circ} \mathrm{C}$. Spores were aseptically harvested and suspended in sterile distilled water with three drops of Tween 80 solution and standardized to a turbidity of $1 \mathrm{McF}$ arland solution $\left(3 \times 10^{8} \mathrm{CFU} / \mathrm{ml}\right)$.

2.4. Determination of Inhibition Concentration. Antifungal activities of the plant extracts were evaluated using the disc diffusion as described by Sigei et al. [22] and according to National committee of clinical and laboratory standards NCCLS now CLSI [23]. The diameters of the inhibition zones produced around the test material were measured with a ruler and recorded in $\mathrm{mm}$. Plant extracts that produced a zone of inhibition of $8-11 \mathrm{~mm}$ were said to be active. Those with zones above $11 \mathrm{~mm}$ were considered very active. Those with zone of inhibition below $8 \mathrm{~mm}$ were considered inactive [24]. The tests were replicated three times for each material.

2.5. Determination of Minimum Inhibitory Concentration. Minimum inhibitory concentration (MIC) was determined through the broth dilution technique. Different concentrations of the extracts were prepared and replicated three times. The extract concentrations were $100 \mathrm{mg} / \mathrm{ml}, 50 \mathrm{mg} / \mathrm{ml}$, and $25 \mathrm{mg} / \mathrm{ml} .5 \mathrm{ml}$ of each concentration of the extract was poured aseptically into a sterile test tube. $1 \mathrm{ml}$ of the toxigenic A. flavus ( 1 McFarland standard) was added. $1 \mathrm{ml}$ of this mixture was poured aseptically into $5 \mathrm{ml}$ of potato dextrose broth (serial dilution) [4]. All the tubes were incubated at $29^{\circ} \mathrm{C}$ for 72 hours. Observations were made for visible fungal growth. The lowest dilution without visible growth for each extract was regarded as the minimum inhibitory concentration

2.6. Treatment of Fungal Spores with Plant Extracts and Light. Concentrations of $450 \mathrm{mg} / \mathrm{ml}$ and $600 \mathrm{mg} / \mathrm{ml}$ of each plant extract were prepared as the working solutions. The $3 \times 10^{8} \mathrm{CFU} / \mathrm{ml}$ McFarland solution was serially diluted up to a concentration of $3 \times 10^{2} \mathrm{CFU} / \mathrm{ml}$. $2 \mathrm{ml}$ of this $\left(3 \times 10^{2} \mathrm{CFU} / \mathrm{ml}\right)$ spore suspension was added to $2 \mathrm{ml}$ of each extract separately. The mixture was well shaken and treated with visible light spectrum at a range of $420 \mathrm{~nm}$ provided by a special lamp from Multiplex Display Fixture [19]. The maximum absorption range of the plant extracts was tested using a spectrophotometer and found to be $420 \mathrm{~nm}$. Irradiation of the plant extracts which were the photosensitizers was done for 10, 20, and 40 minutes. Effects of light irradiated on the photosensitizer (plant extract) for varying time periods (10 mins, 20 mins, and $40 \mathrm{mins}$ ) were tested on the viability of spores of the toxigenic A. flavus. Controls experiments involved adding plant extracts to the conidia suspension without light treatments, reacting conidia without plant extracts with light and conidia without light and plant extracts as described by [19]. Each treatment was replicated three times.

$100 \mathrm{ul}$ of irradiated solution was transferred to PDA plates and incubated at $29^{\circ} \mathrm{C}$. The control experiments were also treated in the same way. Colony forming units (CFUs) were counted after 72 hours of incubation to determine the viability of conidia. 
TABLE 1: Effect of different plant extracts on growth of A. flavus strain UONV003 at different concentrations.

\begin{tabular}{lccc}
\hline Plants & Inhibition zones $(\mathrm{mm}) 600 \mathrm{mg} / \mathrm{ml}$ & Inhibition zones $(\mathrm{mm}) 450 \mathrm{mg} / \mathrm{ml}$ & Inhibition zones $(\mathrm{mm}) 300 \mathrm{mg} / \mathrm{ml}$ \\
\hline S. aculeastrum & $11.92 \pm 0.94^{\mathrm{ab}}$ & $11.00 \pm 0.60^{\mathrm{b}}$ & $11.08 \pm 0.67^{\mathrm{b}}$ \\
S. cordatum & $10.27 \pm 0.32^{\mathrm{bc}}$ & $10.25 \pm 0.52^{\mathrm{b}}$ & $9.50 \pm 0.68^{\mathrm{bc}}$ \\
L. kituiensis & $9.25 \pm 0.70^{\mathrm{c}}$ & $6.92 \pm 0.34^{\mathrm{c}}$ & $6.58 \pm 0.70^{\mathrm{cd}}$ \\
P. africana & $8.50 \pm 0.72^{\mathrm{cd}}$ & $6.00 \pm 0.01^{\mathrm{c}}$ & $3.00 \pm 0.90^{\mathrm{e}}$ \\
O. lamiifolium & $6.42 \pm 0.20^{\mathrm{d}}$ & $6.08 \pm 0.08^{\mathrm{c}}$ & $4.42 \pm 0.96^{\mathrm{de}}$ \\
S. oleracea & $9.25 \pm 0.31^{\mathrm{c}}$ & $7.83 \pm 0.79^{\mathrm{c}}$ & $6.33 \pm 0.99^{\mathrm{c}-\mathrm{e}}$ \\
Positive control $250 \mathrm{mg} / \mathrm{ml}$ & $17.17 \pm 0.40^{\mathrm{a}}$ & $17.17 \pm 0.40^{\mathrm{a}}$ & $17.17 \pm 0.40^{\mathrm{a}}$ \\
Sig $P<0.05$ & 0.00 & 0.00 & 0.00 \\
\hline
\end{tabular}

Numbers are means of twelve replications. One-way Annova was used for analysis and means were separated by Tukey's test. Numbers followed by the same letters in the same column are not significantly different $(P<0.05)$.

TABLE 2: Effect of different plant extracts against growth of A. flavus strain UONV017 at different concentrations.

\begin{tabular}{lccc}
\hline Plants & Inhibition zones $(\mathrm{mm}) 600 \mathrm{mg} / \mathrm{ml}$ & Inhibition zones $(\mathrm{mm}) 450 \mathrm{mg} / \mathrm{ml}$ & Inhibition zones $(\mathrm{mm}) 300 \mathrm{mg} / \mathrm{ml}$ \\
\hline S. aculeastrum & $18.50 \pm 0.71^{\mathrm{a}}$ & $14.42 \pm 0.83^{\mathrm{b}}$ & $11.67 \pm 0.54^{\mathrm{b}}$ \\
S. cordatum & $17.00 \pm 1.26^{\mathrm{a}}$ & $12.00 \pm 0.52^{\mathrm{bc}}$ & $10.67 \pm 0.54^{\mathrm{bc}}$ \\
L. kituiensis & $11.08 \pm 0.53^{\mathrm{b}}$ & $10.17 \pm 0.68^{\mathrm{cd}}$ & $8.08 \pm 0.47^{\mathrm{cd}}$ \\
P. africana & $8.67 \pm 0.69^{\mathrm{b}}$ & $9.25 \pm 0.71^{\mathrm{d}}$ & $6.83 \pm 1.04^{\mathrm{d}}$ \\
O. lamiifolium & $11.42 \pm 0.34^{\mathrm{b}}$ & $6.67 \pm 0.26^{\mathrm{e}}$ & $6.00 \pm 0.83^{\mathrm{d}}$ \\
S. oleracea & $10.08 \pm 0.36^{\mathrm{b}}$ & $9.00 \pm 0.30^{\mathrm{de}}$ & $6.33 \pm 0.14^{\mathrm{d}}$ \\
Positive control $250 \mathrm{mg} / \mathrm{ml}$ & $22.00 \pm 0.63^{\mathrm{a}}$ & $22.00 \pm 0.63^{\mathrm{a}}$ & $22.00 \pm 0.63^{\mathrm{a}}$ \\
Sig $P<0.05)$ & 0.00 & 0.00 & 0.00 \\
\hline
\end{tabular}

Numbers are means of twelve replications. One-way Annova was used for analysis and means were separated by Tukey's test. Numbers followed by the same letters in the same column are not significantly different $(P<0.05)$.

2.7. Phytochemical Screening of Plant Extracts. The six organic and aqueous plant extracts obtained were subjected to phytochemical screening to determine the presence of bioactive agents like flavonoids, steroids, terpenoids, saponins, tannins, alkaloids, and glycosides. Plant extracts from the stock solution of $800 \mathrm{mg} / \mathrm{ml}$ were used for the phytochemical screening $[4,25]$.

2.8. Statistical Analysis. Data analysis was done using SPSS version 16. Data values were expressed as means \pm standard error. Analysis of variance was used and when $F$ was significant $(P \leq 0.05)$, comparison of means was done using Tukey's test.

\section{Results}

3.1. Effect of Organic and Aqueous Plant Extracts on Growth of Toxigenic A. flavus. The crude organic extracts of five out of the six plants tested exhibited antifungal activity against the growth of toxigenic strains of $A$. flavus. The aqueous extracts did not show significant $(P \leq 0.05)$ antifungal activity because all zones of inhibition diameter were below $8 \mathrm{~mm}$. Solanum aculeastrum and Syzygium cordatum plant extracts at $600 \mathrm{mg} / \mathrm{ml}$ against $A$. flavus UONV017 had inhibition diameters that had no significance difference $(P=0.34$ and $P=0.75)$ and hence compared favorably with the standard antifungal control Apron star $(250 \mathrm{mg} / \mathrm{ml})$ which is a class III Blue Active ingredient containing 20\% thiamethoxam $+20 \%$ metalaxyl-M $+2 \%$ difenoconazole. Solanum aculeastrum organic extract had the highest antifungal activity followed by Syzygium cordatum against both strains of A. flavus (Tables 1 and 2). Apart from S. aculeastrum plant extracts against A. flavus UONV003 which had a higher inhibition diameter at $300 \mathrm{mg} / \mathrm{ml}($ mean $=11.08 \pm 0.67 \mathrm{~mm})$ than at $450 \mathrm{mg} / \mathrm{ml}$ (mean $=11.00 \pm 0.60 \mathrm{~mm}$ ) and $P$. africana leaf extracts against A. flavus strain UONV017, which had a higher inhibition diameter at $450 \mathrm{mg} / \mathrm{ml}($ mean $=9.25 \pm 0.71 \mathrm{~mm})$ than at $600 \mathrm{mg} / \mathrm{ml}$ (mean $=8.67 \pm 0.69 \mathrm{~mm}$ ), all the other extracts had the highest inhibitory activity at the highest concentration $(600 \mathrm{mg} / \mathrm{mL})$ and the lowest antifungal activity was at the lowest concentration $(300 \mathrm{mg} / \mathrm{ml}$ ) (Tables 1 and 2).

Comparison of the activities of the organic plant extracts between both strains (UONV003 and UONV017) of A. flavus showed that $A$. flavus UONV003 had smaller inhibition diameters than A. flavus UONV017 at different concentrations (Figure 1).

Extracts of $S$. aculeastrum had the lowest minimum inhibitory concentration at $25 \mathrm{mg} / \mathrm{ml}$ against A. flavus (UONV017) and at $50 \mathrm{mg} / \mathrm{ml}$ against A. flavus UONV003. Syzygium cordatum had an MIC of $50 \mathrm{mg} / \mathrm{ml}$ on A. flavus UONV017 and $100 \mathrm{mg} / \mathrm{ml}$ on A. flavus UONV003.

3.2. Effect of Aqueous Plant Extracts and Photosensitization on A. flavus UONV017 at Different Concentrations and Different Timings. Interaction between aqueous plants extracts and visible light at $600 \mathrm{mg} / \mathrm{ml}$ was statistically significant $(F=$ 55.80; $\mathrm{DF}=18 ; P \leq 0.05$ ). Different plant extracts fungi suspensions had different counts of CFU after light treatment 


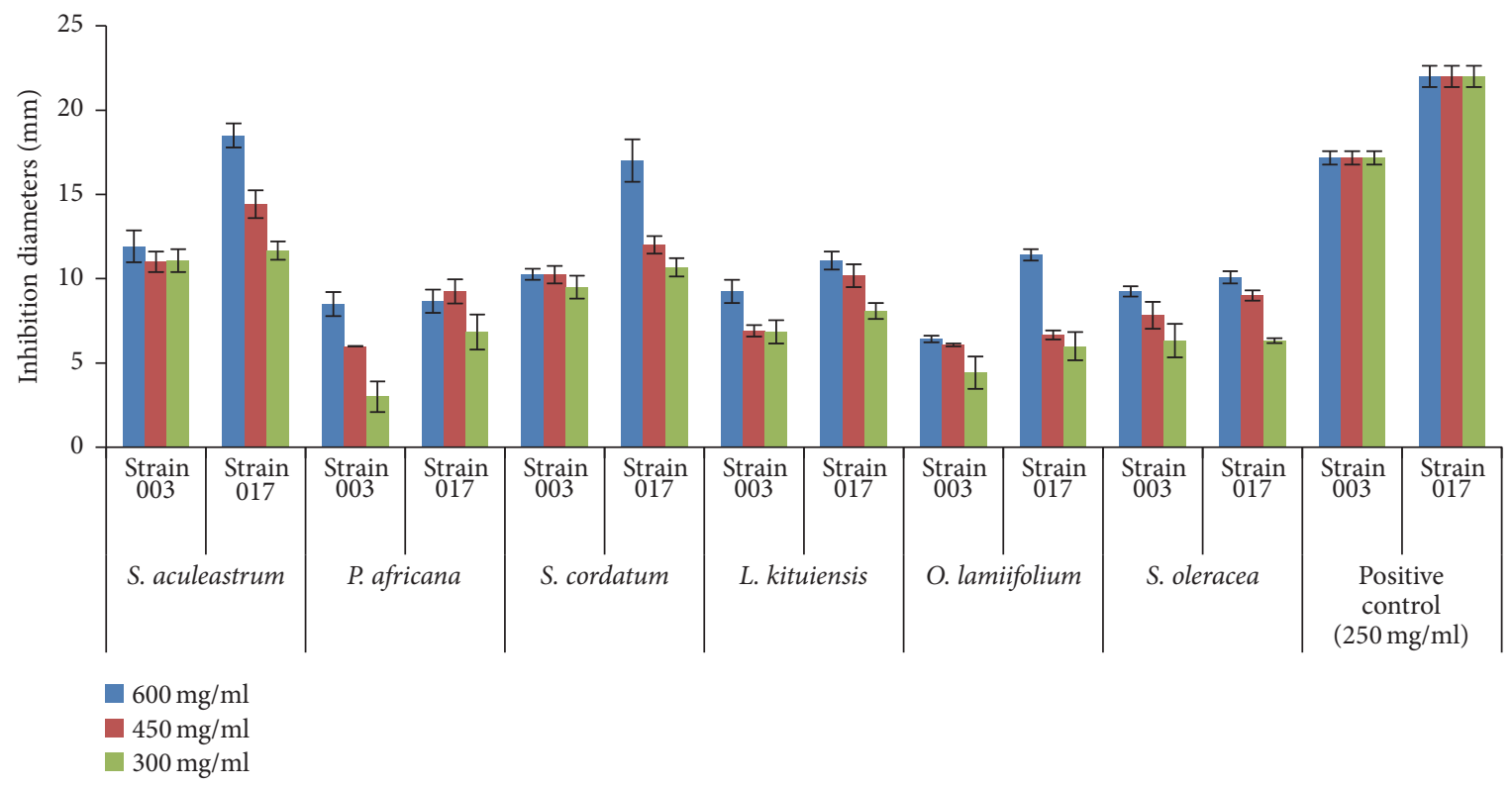

FIGURE 1: Comparison of inhibition of A. flavus UONV017 and that of A. flavus UONV003 by organic plant extracts at different concentrations.

TABLE 3: Effect of aqueous plant extracts and photosensitization on Aspergillus flavus (UONV017).

\begin{tabular}{|c|c|c|c|c|c|c|c|c|}
\hline \multirow{2}{*}{ Plants } & \multicolumn{4}{|c|}{ Colony forming units at $600 \mathrm{mg} / \mathrm{ml}$} & \multicolumn{4}{|c|}{ Colony forming units at $450 \mathrm{mg} / \mathrm{ml}$} \\
\hline & in) & $20(\min )$ & $40(\min )$ & 0 minut & hin) & $20(\min )$ & $40(\min )$ & $0 \mathrm{~min} \mathrm{~s}$ \\
\hline S. aculeastr & $4.00 \pm 1.29^{\mathrm{b}}$ & $4.00 \pm 1.29^{\mathrm{ab}}$ & $2.00 \pm 1.29^{\mathrm{a}}$ & $35 \pm 1.29^{c}$ & $4.00 \pm 1.12^{\mathrm{b}}$ & $4.00 \pm 1.12^{\mathrm{ab}}$ & $3.00 \pm 1.12^{\mathrm{a}}$ & $35 \pm 1.12^{c}$ \\
\hline P. africana & $5.00 \pm 1.29^{\mathrm{b}}$ & $4.00 \pm 1.29^{\mathrm{ab}}$ & $3.00 \pm 1.29^{\mathrm{a}}$ & $71 \pm 1.29^{c}$ & $6.00 \pm 1.12^{\mathrm{b}}$ & $5.00 \pm 1.12^{\mathrm{ab}}$ & $5.00 \pm 1.12^{\mathrm{a}}$ & $71 \pm 1.12^{c}$ \\
\hline S. cordatum & $5.00 \pm 1.29^{\mathrm{b}}$ & $4.00 \pm 1.29^{\mathrm{ab}}$ & $4.00 \pm 1.29^{\mathrm{a}}$ & & $5.00 \pm 1.12^{\mathrm{b}}$ & $4.00 \pm 1.12^{\mathrm{ab}}$ & 4.00 & \\
\hline L. kit & $11.00 \pm 1.29^{\mathrm{b}}$ & $8.00 \pm 1.29^{\mathrm{ab}}$ & $7.00 \pm 1.29^{\mathrm{a}}$ & & $13.00 \pm 1.12^{\mathrm{b}}$ & $11.00 \pm 1.12^{\mathrm{ab}}$ & $10.00 \pm 1.12^{\mathrm{a}}$ & \\
\hline O. lamiifolium & $12.00 \pm 1.29^{\mathrm{b}}$ & $8.00 \pm 1.29^{\mathrm{ab}}$ & $6.00 \pm 1$ & & & & $10.00 \pm 1.12^{\mathrm{a}}$ & \\
\hline S. oleracea & $13.00 \pm 1.29^{\mathrm{b}}$ & $10.00 \pm 1.29^{\mathrm{ab}}$ & $7.00 \pm$ & $9^{c}$ & $12.00 \pm$ & $11.00 \pm$ & $10.00 \pm 1.12^{\mathrm{a}}$ & $54 \pm$ \\
\hline 0 extracts (ctrl) & $76.00 \pm 1.29^{\mathrm{ab}}$ & $73.00 \pm 1.29^{\mathrm{b}}$ & $73.00 \pm 1.29^{b}$ & $80.00 \pm 1.29^{\mathrm{a}}$ & $76.00 \pm 1.12^{\mathrm{ab}}$ & $73.00 \pm 1.12^{\mathrm{b}}$ & $73.00 \pm 1.12^{b}$ & $80.00 \pm 1.12^{\mathrm{a}}$ \\
\hline
\end{tabular}

Numbers are means of three replications. Two-way Annova was used for analysis and means were separated by Tukey's test. Numbers followed by the same letters in the same row within each concentration are not significantly different $(P<0.05)$.

at varying time durations. Solanum aculeastrum (mean $=2$ CFUs) had the lowest CFU reading at 40 minutes and hence was the most effective. Other than the controls, S. oleracea (mean $=13$ CFUs) had the highest CFU reading at 10 minutes and hence was the least effective at $600 \mathrm{mg} / \mathrm{ml}$. At $450 \mathrm{mg} / \mathrm{ml}$, interaction between the aqueous extracts and light caused significant $(F=71.46$; $\mathrm{df}=18 ; P \leq 0.05)$ reduction of CFUs. Solanum aculeastrum (mean $=3$ CFUs) was the most effective with the lowest number of CFUs while O. lamiifolium (mean $=14$ CFUs) was the least effective with the highest number of CFUs at 10 minutes.

Comparison of photosensitization activities within different time durations proved that treatments kept under light for the highest duration of time (40 minutes) had the lowest CFU counts, hence proving the highest inactivation of fungal spores. Treatments that were under light for the shortest time duration (10 minutes) exhibited a higher number of CFUs; samples with no light and no extract treatment had the highest CFU count (Table 3).
3.3. Effect of Aqueous Plant Extracts and Photosensitization on A. flavus (UONV003) at Different Concentrations and Different Timings. Statistically significant $(F=31.21$; DF $=$ $18 ; P \leq 0.05)$ interaction existed between aqueous plants extracts and light in their activity against $A$. flavus strain UONV003 at $600 \mathrm{mg} / \mathrm{ml}$. Syzygium cordatum (mean $=3$ CFUs) had the lowest CFU reading at 40 minutes and hence was the most effective. Lippia kituiensis (mean =16 CFUs) had the highest CFU reading at 10 minutes and hence was the least effective. At $450 \mathrm{mg} / \mathrm{ml}$, interaction between aqueous extracts and light was also significant $(F=35.86 ; \mathrm{DF}=18 ; P \leq 0.05)$. Solanum aculeastrum (mean $=3$ CFUs) was the most effective while L. kituiensis (mean =17 CFUs) was the least effective at 10 minutes (Table 4).

3.4. Effect of Organic Plant Extracts and Photosensitization on A. flavus (UONV017) at Different Concentrations and Different Timings. Organic extracts at both concentrations of $600 \mathrm{mg} / \mathrm{ml}$ displayed significant $(F=32.72 ; \mathrm{DF}=18$; 
TABLE 4: Effect of aqueous plant extracts and photosensitization on Aspergillus flavus UONV003.

\begin{tabular}{|c|c|c|c|c|c|c|c|c|}
\hline \multirow{2}{*}{ Plants } & \multicolumn{4}{|c|}{ Colony forming units at $600 \mathrm{mg} / \mathrm{ml}$} & \multicolumn{4}{|c|}{ Colony forming units at $450 \mathrm{mg} / \mathrm{ml}$} \\
\hline & in) & $20(\min )$ & $40(\min )$ & 0 minute & (n) & $20(\min )$ & $40(\min )$ & $0 \mathrm{minu}$ \\
\hline & $5.00 \pm 1.70^{\mathrm{b}}$ & $4.00 \pm 1.70^{\mathrm{ab}}$ & $4.00 \pm 1.70^{\mathrm{a}}$ & $43 \pm 1.70^{c}$ & $5.00 \pm 1.60^{\mathrm{b}}$ & $4.00 \pm 1.60^{\mathrm{ab}}$ & $4.00 \pm 1.60^{\mathrm{a}}$ & $43 \pm 1.60^{c}$ \\
\hline P. africt & $6.00 \pm 1.70^{\mathrm{b}}$ & $5.00 \pm 1.70^{\mathrm{ab}}$ & $4.00 \pm 1.70^{\mathrm{a}}$ & $72 \pm 1.70^{c}$ & $5.00 \pm 1.60^{\mathrm{b}}$ & $4.00 \pm 1.60^{\mathrm{ab}}$ & $4.00 \pm 1.60^{\mathrm{a}}$ & $72 \pm 1.60^{c}$ \\
\hline$u m$ & $5.00 \pm 1.70^{\mathrm{b}}$ & $4.00 \pm 1.70^{\mathrm{ab}}$ & $3.00 \pm 1.70^{\mathrm{a}}$ & & $5.00 \pm 1.60^{\mathrm{b}}$ & $4.00 \pm 1.60^{\mathrm{ab}}$ & $3.00 \pm 1.60^{\mathrm{a}}$ & $34 \pm 1.60^{c}$ \\
\hline L. $k$ & $16.00 \pm 1.70^{\mathrm{b}}$ & $12.00 \pm 1.70^{\mathrm{ab}}$ & $8.00 \pm 1.70^{\mathrm{a}}$ & & $17.00 \pm 1.60^{\mathrm{b}}$ & $14.00 \pm 1.60^{\mathrm{ab}}$ & $12.00 \pm 1.60^{\mathrm{a}}$ & \\
\hline folium & $13.00 \pm 1.70^{\mathrm{b}}$ & & $8.00 \pm 1.70^{\mathrm{a}}$ & & & $11.00 \pm 1.60^{\mathrm{ab}}$ & $10.00 \pm 1.60^{\mathrm{a}}$ & \\
\hline S. oleracea & $14.00 \pm 1.70^{\mathrm{b}}$ & $14.00 \pm 1.70^{\mathrm{ab}}$ & $11.00 \pm 1.70^{\mathrm{a}}$ & $70^{c}$ & $16.00 \pm 1.60^{\mathrm{b}}$ & $13.00 \pm 1.60^{\mathrm{ab}}$ & $10.00 \pm 1.60^{\mathrm{a}}$ & $58 \pm$ \\
\hline 0 extracts (ctrl) & $76.00 \pm 1.70^{\mathrm{a}}$ & $76.00 \pm 1.70^{\mathrm{a}}$ & $74.00 \pm 1.70^{\mathrm{a}}$ & $81.00 \pm 1.70^{\mathrm{a}}$ & $76.00 \pm 1.60^{\mathrm{a}}$ & $76.00 \pm 1.60^{\mathrm{a}}$ & $74.00 \pm 1.60^{\mathrm{a}}$ & $81.00 \pm 1.60^{\mathrm{a}}$ \\
\hline
\end{tabular}

Numbers are means of three replications. Two-way Annova was used for analysis and means were separated by Tukey's test. Numbers followed by the same letters in the same row within each concentration are not significantly different $(P<0.05)$.

TABLE 5: Effect of organic plant extracts and photosensitization on Aspergillus flavus (UONV017).

\begin{tabular}{|c|c|c|c|c|c|c|c|c|}
\hline \multirow{2}{*}{ Plants } & \multicolumn{4}{|c|}{ Colony forming units at $600 \mathrm{mg} / \mathrm{ml}$} & \multicolumn{4}{|c|}{ Colony forming units at $450 \mathrm{mg} / \mathrm{ml}$} \\
\hline & $10(\min )$ & $20(\min )$ & $40(\min )$ & 0 minutes $(\mathrm{ctrl})$ & $10(\min )$ & $20(\min )$ & $40(\min )$ & 0 minutes $(\mathrm{ctrl})$ \\
\hline S. aculeastrum & $11.00 \pm 1.7^{\mathrm{b}}$ & $11.00 \pm 1.7^{\mathrm{a}}$ & $8.00 \pm 1.7^{\mathrm{a}}$ & $62 \pm 1.71^{c}$ & $24.00 \pm 1.73^{\mathrm{b}}$ & $12.00 \pm 1.73^{\mathrm{a}}$ & $9.00 \pm 1.7^{\mathrm{a}}$ & $62 \pm 1.73^{c}$ \\
\hline P. africana & $10.00 \pm 1.7^{\mathrm{b}}$ & $10.00 \pm 1.7^{\mathrm{a}}$ & $8.00 \pm 1.7^{\mathrm{a}}$ & $76 \pm 1.71^{\mathrm{c}}$ & $41.00 \pm 1.73^{\mathrm{b}}$ & $23.00 \pm 1.73^{\mathrm{a}}$ & $14.00 \pm 1.73^{\mathrm{a}}$ & $76 \pm 1.73^{c}$ \\
\hline S. cordatum & $10.00 \pm 1.7^{\mathrm{b}}$ & $8.00 \pm 1.71^{\mathrm{a}}$ & $4.00 \pm 1.7^{\mathrm{a}}$ & $51 \pm 1.71^{\mathrm{c}}$ & $11.00 \pm 1.73^{\mathrm{b}}$ & $8.00 \pm 1.73^{\mathrm{a}}$ & $7.00 \pm 1.73^{\mathrm{a}}$ & $51 \pm 1.73^{c}$ \\
\hline L. kituiensis & $16.00 \pm 1.7^{\mathrm{b}}$ & $12.00 \pm 1.7^{\mathrm{a}}$ & $8.00 \pm 1.7^{\mathrm{a}}$ & $44 \pm 1.71^{\mathrm{c}}$ & $22.00 \pm 1.73^{\mathrm{b}}$ & $12.00 \pm 1.73^{\mathrm{a}}$ & $11.00 \pm 1.73^{\mathrm{a}}$ & $48 \pm 1.73^{\mathrm{c}}$ \\
\hline O. lamiifolium & $19.00 \pm 1.7^{\mathrm{b}}$ & $12.00 \pm 1.7^{\mathrm{a}}$ & $9.00 \pm 1.7^{\mathrm{a}}$ & $48 \pm 1.71^{\mathrm{c}}$ & $23.00 \pm 1.73^{\mathrm{b}}$ & $16.00 \pm 1.73^{\mathrm{a}}$ & $14.00 \pm 1.73^{\mathrm{a}}$ & $72 \pm 1.73^{c}$ \\
\hline S. oleracea & $11.00 \pm 1.7^{\mathrm{b}}$ & $10.00 \pm 1.7^{\mathrm{a}}$ & $9.00 \pm 1.7^{\mathrm{a}}$ & $47 \pm 1.71^{c}$ & $14.00 \pm 1.73^{\mathrm{b}}$ & $12.00 \pm 1.73^{\mathrm{a}}$ & $9.00 \pm 1.73^{\mathrm{a}}$ & $47 \pm 1.73^{c}$ \\
\hline 0 extracts (ctrl) & $76.00 \pm 1.7^{\mathrm{ab}}$ & $73.00 \pm 1.7^{\mathrm{b}}$ & $73.00 \pm 1.1^{\mathrm{b}}$ & $80.00 \pm 1.71^{\mathrm{a}}$ & $76.00 \pm 1.73^{b}$ & $73.00 \pm 1.73^{\mathrm{b}}$ & $73.00 \pm 1.73^{b}$ & $80.00 \pm 1.73^{\mathrm{ab}}$ \\
\hline
\end{tabular}

Numbers are means of three replications. Two-way Annova was used for analysis and means were separated by Tukey's test. Numbers followed by the same letters in the same row within each concentration are not significantly different $(P<0.05)$.

$P \leq 0.05)$ photosensitization activity. Syzygium cordatum (mean $=4$ CFUs) had the lowest CFU reading at 40 minutes at a concentration of $600 \mathrm{mg} / \mathrm{ml}$. Ocimum lamiifolium (mean $=19$ CFUs) had the highest CFU reading at 10 minutes at $600 \mathrm{mg} / \mathrm{ml}$. At $450 \mathrm{mg} / \mathrm{ml}$, there was also significant $(F=$ 23.615; $\mathrm{DF}=18 ; P \leq 0.05)$ photosensitization activity. Syzygium cordatum (mean $=7$ CFUs) was the most effective at 40 minutes (Table 5).

\subsection{Effect of Aqueous Plant Extracts and Photosensitization} on A. flavus (UONV003) at Different Concentrations and Different Timings. Interaction between organic plants extracts and light was statistically significant $(F=32.97$; DF = $18 ; P \leq 0.05)$ at a concentration of $600 \mathrm{mg} / \mathrm{ml}$. Solanum aculeastrum (mean $=9$ CFUs) had the lowest CFU reading at 40 minutes. Lippia kituiensis (mean $=23$ CFUs) had the highest $\mathrm{CFU}$ reading at 10 minutes and hence was the least effective at $600 \mathrm{mg} / \mathrm{ml}$. At $450 \mathrm{mg} / \mathrm{ml}$, organic extracts exhibited significant $(F=19.39 ; \mathrm{DF}=18 ; P \leq 0.05)$ photosensitization activity. Solanum aculeastrum (mean $=9$ CFUs) was still the most effective at 40 minutes (Table 6).

Comparison of photosensitization effects between aqueous and organic extracts proved that both extracts were effective against toxigenic $A$. flavus (UONV017 and UONV003) because there was significant reduction of CFU in comparison with the controls. Aqueous extracts had a lower number of CFUs than the organic extracts. The higher concentration
$(600 \mathrm{mg} / \mathrm{ml})$ had greater reduction of CFU compared to the $450 \mathrm{mg} / \mathrm{ml}$ (Table 6).

3.6. Phytochemical Screening of Selected Medicinal Plant Extracts. Six different bioactive compounds, namely, saponins, flavonoids, terpenoids, tannins, alkaloids, and glycosides, were found in the plant extracts during this study. Spinacia oleracea plant extracts had the highest percentage (21.6), followed by S. aculeastrum (19.6\%), while Prunus africana had the lowest percentage (9.8) of bioactive compounds. Flavonoids had the highest frequency $(21.6 \%)$ while terpenoids and steroids had the lowest frequency (9.8\%). Organic extracts had a higher frequency $(60.8 \%)$ of bioactive compounds compared to aqueous extracts (39.2\%) (Table 7).

\section{Discussions}

This study revealed that the selected medicinal plant extracts had antifungal activity against toxigenic A. flavus. Five out of the six organic plants extracts assayed indicated antifungal activity which varied depending on concentration and plant species. At $600 \mathrm{mg} / \mathrm{ml}$, the antifungal activity of $S$. aculeastrum and $S$. cordatum had no significant difference with the standard antifungal control Apron star. This reveals the significance of crude plant extracts in lowering risk of toxigenic A. flavus, hence lowering aflatoxin contamination [26]. 
TABLE 6: Effect of organic plant extracts and photosensitization on Aspergillus flavus UONV003.

\begin{tabular}{|c|c|c|c|c|c|c|c|c|}
\hline \multirow{2}{*}{ Plants } & \multicolumn{4}{|c|}{ Colony forming units at $600 \mathrm{mg} / \mathrm{ml}$} & \multicolumn{4}{|c|}{ Colony forming units at $450 \mathrm{mg} / \mathrm{ml}$} \\
\hline & 10 (min) & 20 (min) & $40(\min )$ & 0 minutes (ctrl) & 10 (min) & $20(\min )$ & $40(\min )$ & 0 minutes (ctrl) \\
\hline S. aculeastrum & $12.00 \pm 1.7^{\mathrm{b}}$ & $12.00 \pm 1.76^{\mathrm{b}}$ & $9.00 \pm 1.76^{\mathrm{a}}$ & $69 \pm 1.76^{c}$ & $26.00 \pm 2.22^{b}$ & $13.00 \pm 2.22^{\mathrm{a}}$ & $9.00 \pm 2.22^{\mathrm{a}}$ & $69 \pm 2.22^{c}$ \\
\hline P. africana & $18.00 \pm 1.7^{\mathrm{b}}$ & $17.00 \pm 1.76^{\mathrm{b}}$ & $11.00 \pm 1.7^{\mathrm{a}}$ & $77 \pm 1.76^{\mathrm{c}}$ & $34.00 \pm 2.22^{\mathrm{b}}$ & $20.00 \pm 2.22^{\mathrm{a}}$ & $15.00 \pm 2.2^{\mathrm{a}}$ & $77 \pm 2.22^{c}$ \\
\hline S. cordatum & $15.00 \pm 1.7^{\mathrm{b}}$ & $14.00 \pm 1.76^{\mathrm{b}}$ & $10.00 \pm 1.7^{\mathrm{a}}$ & $60 \pm 1.76^{\mathrm{c}}$ & $18.00 \pm 2.22^{\mathrm{b}}$ & $15.00 \pm 2.22^{\mathrm{a}}$ & $12.00 \pm 2.2^{\mathrm{a}}$ & $60 \pm 2.22^{c}$ \\
\hline L. kituiensis & $23.00 \pm 1.7^{\mathrm{b}}$ & $12.00 \pm 1.76^{\mathrm{b}}$ & $14.00 \pm 1.7^{\mathrm{a}}$ & $64 \pm 1.76^{\mathrm{c}}$ & $27.00 \pm 2.22^{\mathrm{b}}$ & $13.00 \pm 2.22^{\mathrm{a}}$ & $11.00 \pm 2.2^{\mathrm{a}}$ & $74 \pm 2.22^{c}$ \\
\hline O. lamiifolium & $12.00 \pm 1.7^{\mathrm{b}}$ & $10.00 \pm 1.76^{\mathrm{b}}$ & $11.00 \pm 1.7^{\mathrm{a}}$ & $74 \pm 1.76^{\mathrm{c}}$ & $13.00 \pm 2.22^{\mathrm{b}}$ & $10.00 \pm 2.22^{\mathrm{a}}$ & $10.00 \pm 2.2^{\mathrm{a}}$ & $52 \pm 2.22^{c}$ \\
\hline S. oleracea & $12.00 \pm 1.7^{\mathrm{b}}$ & $12.00 \pm 1.7^{\mathrm{b}}$ & $10.00 \pm 1.7^{\mathrm{a}}$ & $52 \pm 1.76^{\mathrm{c}}$ & $11.00 \pm 2.22^{\mathrm{b}}$ & $11.00 \pm 2.22^{\mathrm{a}}$ & $10.00 \pm 2.2^{\mathrm{a}}$ & $47 \pm 2.22^{c}$ \\
\hline 0 extracts (ctrl) & $76.00 \pm 1.7^{\mathrm{a}}$ & $76.00 \pm 1.76^{\mathrm{a}}$ & $74.00 \pm 1.7^{\mathrm{a}}$ & $81.00 \pm 1.7^{\mathrm{a}}$ & $76.00 \pm 2.22^{\mathrm{a}}$ & $76.00 \pm 2.22^{\mathrm{a}}$ & $74.00 \pm 2.2^{\mathrm{a}}$ & $81.00 \pm 2.2^{\mathrm{a}}$ \\
\hline
\end{tabular}

Numbers are means of three replications. Two-way Annova was used for analysis and means were separated by Tukey's test. Numbers followed by the same letters in the same row within each concentration are not significantly different $(P<0.05)$.

TABLE 7: Frequency of bioactive compounds in plant extracts.

\begin{tabular}{lccc}
\hline & Frequency & Percent & Cumulative percent \\
\hline Plants & 5 & 9.8 & 9.8 \\
Prunus africana & 7 & 13.7 & 23.5 \\
Lippia kituiensis & 10 & 19.6 & 43.1 \\
Solanum aculeastrum & 9 & 17.6 & 60.8 \\
Syzygium cordatum & 11 & 21.6 & 82.4 \\
Spinacia oleracea & 9 & 17.6 & 100 \\
Ocimum lamiifolium & $\mathbf{5 1}$ & $\mathbf{1 0 0}$ & 15.7 \\
Total & & & 33.3 \\
Bioactive compounds & 8 & 15.7 & 54.9 \\
Saponins & 9 & 17.6 & 74.5 \\
Tannins & 11 & 21.6 & 90.2 \\
Flavonoids & 10 & 1.96 & 100 \\
Alkaloids & 8 & 15.7 & 9.8 \\
Glycosides & 5 & $\mathbf{1 0 0}$ & \\
Terpenoids and steroids & $\mathbf{5 1}$ & & \\
Total & & 60.8 & \\
Formulation & 31 & 39.2 & $\mathbf{1 0 0}$ \\
Organic & 20 & $\mathbf{5 1}$ & \\
Aqueous & & & \\
Total & & & \\
\hline
\end{tabular}

Organic extracts exhibited significant antifungal activity while the aqueous extracts did not. The organic extracts had a higher percentage of bioactive compounds $(60.8 \%)$ than the aqueous extracts (39.2\%). Different solvents have varying levels of solubility for different bioactive compounds. The reason for this sequence in activity may be that bioactive compounds that cause the antimicrobial activity dissolve easily in organic compared to aqueous solvents [27]. This supports earlier findings that organic leaf extracts had stronger antimicrobial activity compared to aqueous extracts [28]. The antifungal activity was directly proportional to the concentration of the plant extracts; the highest concentration $(600 \mathrm{mg} / \mathrm{ml})$ had the largest inhibition diameters while the lowest concentration $(300 \mathrm{mg} / \mathrm{ml})$ had the smallest inhibition diameters. This could be explained by the fact that the high concentration contains a higher percentage of the bioactive compounds.
This observation parallels findings in a study carried out by Mahmoud et al. [29] and Kiswii et al. [4]. Phytochemical screening indicated presence of tannins, flavonoids, alkaloids, glycosides, steroids, and saponins in varying proportions in the plant extracts. The antifungal efficacy of plant extracts in this study could be associated with the bioactive compounds indicated by the phytochemical analysis. Organic fruit extracts of $S$. aculeastrum had the best antifungal activity against both strains of toxigenic A. flavus. Solanum aculeastrum in our study contained ten bioactive compounds which is $19.6 \%$ according to the phytochemical screening. These could be the possible cause of the antifungal activity. The glycosides, alkaloids, and saponins in S. aculeastrum fruits have been associated with anticancer (skin and cervix), anti-inflammatory, and anticholesterol activities [30]. This agrees with the results of [31] where organic and aqueous 
fruit extracts of S. aculeastrum were tested against fungi and bacteria and they had good antimicrobial activity although antifungal activity caused by organic extracts was higher than that caused by aqueous extracts. Syzygium cordatum bark extracts had significant antifungal activity against both A. flavus strains. This agrees with [32] where S. cordatum bark (organic extract) exhibited antifungal activity. In this study, S. cordatum contains nine bioactive compounds which are $17.6 \%$. These as supported by [33] were the causes of antifungal activity. Syzygium cordatum at a concentration of $450 \mathrm{mg} / \mathrm{ml}$ had higher inhibition diameter compared to that of $600 \mathrm{mg} / \mathrm{ml}$. This could have been caused by the thickness of the higher concentration which interfered with diffusion of the extract and hence a lower diameter of inhibition.

Organic extracts of $P$. africana, L. kituiensis, S. oleracea, and $O$. lamiifolium had antifungal activity against both strains of A. flavus. They all had varying levels of bioactive compounds which were attributed to the varying levels of antifungal activity and this justifies the use of these plant extracts in ethnomedicine. The percentage of bioactive compounds (9.8\%) in P. africana was the lowest and this could be attributed to the low antifungal activity by this extract. Organic extracts of $P$. africana against $A$. flavus UONV017 had higher antifungal activity at $450 \mathrm{mg} / \mathrm{ml}$ than at $600 \mathrm{mg} / \mathrm{ml}$. This could have been caused by thickness in the higher concentration which interfered with diffusion. Spinacia oleracea extracts had the highest percentage of bioactive compounds (21.6\%). Despite having the highest percentage of bioactive compounds, it did not prove the highest level of antifungal activity. This could be attributed to presence of the bioactive compounds in small amounts. Studies by $[28,34,35]$ have proved that bioactive compounds in these plant extracts cause them to have antifungal activity.

The bioactive compounds detected in this study have been shown to cause antimicrobial activities in other studies through various mechanisms [27, 36-38]. Increase in the concentration of bioactive compounds increases the antifungal activity as reported by [39]. This supports the increase in antifungal activity caused by increase in plant extract concentration in our study.

Solanum aculeastrum that had the highest antifungal activity recorded the lowest MIC values for both strains. The low MIC could be attributed to the enhanced antifungal activity. Kiswii et al. [4] found out that the extracts with the highest antifungal activity had the lowest MIC value.

Strain UONV003 of A. flavus proved to be more resistant compared to A. flavus UONV017 in terms of inhibition diameters and also MIC though both are toxigenic strains. This could be explained by the fact that pathogenicity varies between different strains of $A$. flavus and strains with higher pathogenicity may exhibit higher resistance. This parallels a study that was carried out to test pathogenicity and toxigenicity of ground nut $A$. flavus strains. Results showed that there was variation in pathogenicity within the same level of toxigenic strains [40].

This study found that both aqueous and organic plant extracts in combination with light inactivated A. flavus spores, though the former were more effective. Syzygium cordatum which had significant inhibition even in the aqueous form against both strains have been reported to have several antimicrobial abilities [32]. Solanum aculeastrum was the second most effective against both strains in the organic form and the most effective in the aqueous form against both strains. Solanum aculeastrum possess several antimicrobial activities and of significance to this study; the fruit extracts possess antifungal activity against Aspergillus spp. [30].

Both aqueous and organic extracts of the other four plants, O. lamiifolium, S. oleracea, P. africana, and L. kituiensis, had significant reduction of spores as they had low CFU counts compared to the controls against both strains. All the named plants have been reported to possess several antimicrobial activities [28, 41-43]. The higher CFU count in the three controls is an indicator that the visible light plant extract combination was the major cause of conidia inactivation and not light alone or plant extracts alone.

The photo degrading effect of plant extracts in this study is supported by a study where viability Penicillium digitatum conidia was tested using blue light and a dye (ERY) acting as a photosensitizer. Blue light alone or the photosensitizer alone did not reduce the viability of the nongerminated conidia as compared to the conidia viability control. The control was comprised of no light and no photosensitizer treatment. However, nongerminated conidia treated with light and photosensitizer significantly reduced colony forming units (CFUs) by 40 and $70 \%$ with blue light of 80 and $100 \mathrm{~J} / \mathrm{cm}^{2}$, respectively, compared to control [44]. In another study, plant extracts that affect the central nervous system were tested for photoprotection and photosensitization. They were tested at wavelengths ranging from $280 \mathrm{~nm}$ to $436 \mathrm{~nm}$. The plants showed photo protection effect at low concentration and photosensitization effects at the higher concentrations [45]. This also parallels this study where the photosensitization effect was directly proportional to the concentration of the plant extracts. This is explained by the fact that higher concentrations have high number of bioactive compounds which are reactivated by light to inactivate toxigenic microbes.

Photosensitization effect is attributed to the contents of the plant extracts which have strong absorption at high wavelength range [45]. Disinfection of water using the solar energy and plant extracts has been proven. Photodynamic activity was attributed to the presence of quinines and anthraquinones which generate singlet oxygen killing the microorganisms in the water. Two $\mathrm{ml}$ of plant extract per one litre of the polluted water was exposed to the solar energy for one hour to allow complete inactivation of the coli forms [46]. This parallels our study where plant extracts in combination with toxigenic molecules are exposed to light for a specific duration leading to reduced fungal growth.

In this study, the time of exposure of the A. flavus and plant extract under light was indirectly proportional to the CFU counts; this indicates that more light exposure led to more spore knockout. This parallels a study by [19] where increase in light dose increased the rate of spore knockout. In this study also, spore reduction was higher at the higher plant extract concentration of $600 \mathrm{mg} / \mathrm{ml}$ compared to the $450 \mathrm{mg} / \mathrm{ml}$. In the study by [19], rate of spore knockout increased with the extract concentrations up to a certain level where the rate of spore knockout decreased with the 
highest concentration. This was attributed to the high fluid turbidity which may have caused reduced light penetration and transmission. In this study, therefore, the extract concentrations were at a suitable turbidity which did not cause much inhibition of light penetration and inhibition.

Both aqueous and organic extracts were effective photosensitizers though aqueous extracts had a greater reduction of spores than the organic extracts. This is supported by a study by [19] where both organic and aqueous extracts were effective though the aqueous extracts had better activity.

Extracts used in this study have not been tested for antifungal activity and photosensitization against toxigenic $A$. flavus in another study. The findings of this study, therefore, could fulfill the need of new antifungal structure due to unavailability of effective antifungal agents against toxigenic A. flavus, resistance of the fungi to the available methods, and the shortcomings of these methods [46]. The plants in the selected region were correlated with the biodiversity of the region and they are accessible for research and new developments. Plant extracts are of key importance because bioactive compounds in higher plants are biodegradable and selectively toxic.

\section{Conclusion and Recommendations}

In this study, the medicinal plant extracts were found effective against conidia of toxigenic A. flavus. Organic extracts had greater antifungal activity. This makes a good background for research on aflatoxins because aflatoxins also dissolve better in organic extracts compared to aqueous ones. The extracts were found to contain bioactive compounds which were the specific causes of antifungal activity. Isolation of these useful bioactive compounds using the guidance of phytochemical results should be done. The bioactive compounds should be produced in larger quantities for use in the control of the toxigenic fungi. The consumable plant extract Spinacia oleracea in this study can act as a good candidate to treat food and feed which can then be dried under sunlight before storage leading to inactivation and death of the toxigenic $A$. flavus conidia. This is a viable technique to control aflatoxin contamination in storage because consumption of the dead A. flavus has no health implications. This could be applied to all the plant extracts in this study after a toxicity test to ensure safety.

\section{Conflicts of Interest}

The authors declare that they have no conflicts of interest.

\section{Acknowledgments}

The authors would like to acknowledge the University of Nairobi for funding this research through a scholarship.

\section{References}

[1] S. S. Hua, Biocontrol of Aspergillus flavus, vol. 2, Pichia anomala, 2013.
[2] M. E. Zain, "Impact of mycotoxins on humans and animals," Journal of Saudi Chemical Society, vol. 15, no. 2, pp. 129-144, 2011.

[3] S. Okoth, B. Nyongesa, V. Ayugi, E. Kan'gethe, H. Korhonen, and V. Joutsjoki, "Toxigenic potential of Aspergillus species occurring on maize kernels from two Agro-Ecological zones in Kenya," Toxins, vol. 4, no. 11, pp. 991-1007, 2012.

[4] T. M. Kiswii, E. O. Monda, P. O. Okemo, C. Bii, and A. E. Alakonya, "Efficacy of selected medicinal plants from eastern kenya against Aspergillus flavus," Journal of Plant Sciences, vol. 2, no. 5, pp. 226-231, 2014.

[5] J. I. Pitt, “Toxigenic fungi and mycotoxins," British Medical Bulletin, vol. 56, no. 1, pp. 184-192, 2000.

[6] E. G. Lizárraga-Paulín, E. Moreno-Martínez, and S. P. MirandaCastro, Aflatoxins and their impact on human and animal health: an emerging problem, INTECH Open Access Publisher, 2011.

[7] S. M. Herzallah, "Aflatoxin B1 residues in eggs and flesh of laying hens fed aflatoxin B1 contaminated diet," American Journal of Agricultural and Biological Science, vol. 8, no. 2, pp. 156-161, 2013.

[8] C. Probst, F. Schulthess, and P. J. Cotty, "Impact of Aspergillus section Flavi community structure on the development of lethal levels of aflatoxins in Kenyan maize (Zea mays)," Journal of Applied Microbiology, vol. 108, no. 2, pp. 600-610, 2010.

[9] G. S. Shephard, "Impact of mycotoxins on human health in developing countries," Food Additives and Contaminants, vol. 25 , no. 2, pp. 146-151, 2008.

[10] C. Njuguna, Making Kenyan maize safe from deadly aflatoxins, Science in Africa, 2010.

[11] S. Thippeswamy, D. C. Mohana, R. U. Abhishek, and K. Manjunath, "Inhibitory activity of plant extracts on aflatoxin B1 biosynthesis by Aspergillus flavus," Journal of Agricultural Science and Technology, vol. 16, no. 5, pp. 1123-1132, 2014.

[12] K. C. Ehrlich, "Non-aflatoxigenic Aspergillus flavus to prevent aflatoxin contamination in crops: advantages and limitations," Global health issues of aflatoxins in food and agriculture: Challenges and opportunities, vol. 25, 2015.

[13] J. Köhl, J. Postma, P. Nicot, M. Ruocco, and B. Blum, "Stepwise screening of microorganisms for commercial use in biological control of plant-pathogenic fungi and bacteria," Biological Control, vol. 57, no. 1, pp. 1-12, 2011.

[14] T. Murugan, J. A. Wins, and M. Murugan, "Antimicrobial activity and phytochemical constituents of leaf extracts of cassia auriculata," Indian Journal of Pharmaceutical Sciences, vol. 75, no. 1, p. 122, 2013.

[15] P. A. Paranagama, K. H. T. Abeysekera, K. Abeywickrama, and L. Nugaliyadde, "Fungicidal and anti-aflatoxigenic effects of the essential oil of Cymbopogon citratus (DC.) Stapf. (lemongrass) against Aspergillus flavus Link. isolated from stored rice," Letters in Applied Microbiology, vol. 37, no. 1, pp. 86-90, 2003.

[16] M. M. Kaigongi, S. F. Dossaji, J. M. Nguta, C. W. Lukhoba, and F. M. Musila, "Antimicrobial activity, toxicity and phytochemical screening of four medicinal plants traditionally used in Msambweni district, Kenya," Journal of Biology Agriculture and Healthcare, vol. 4, no. 28, 2014.

[17] H. D. de Menezes, G. B. Rodrigues, S. D. P. Teixeira et al., "In vitro photodynamic inactivation of plant-pathogenic fungi Colletotrichum acutatum and Colletotrichum gloeosporioides with novel phenothiazinium photosensitizers," Applied and Environmental Microbiology, vol. 80, no. 5, pp. 1623-1632, 2014. 
[18] W. W. Jong, P. J. Tan, F. A. Kamarulzaman et al., "Photodynamic activity of plant extracts from Sarawak, Borneo," Chemistry and Biodiversity, vol. 10, no. 8, pp. 1475-1486, 2013.

[19] B. A. Temba, M. T. Fletcher, G. P. Fox, J. J. W. Harvey, and Y. Sultanbawa, "Inactivation of Aspergillus flavus spores by curcumin-mediated photosensitization," Food Control, vol. 59, pp. 708-713, 2016.

[20] K. M. Thembo, H. F. Vismer, N. Z. Nyazema, W. C. A. Gelderblom, and D. R. Katerere, "Antifungal activity of four weedy plant extracts against selected mycotoxigenic fungi," Journal of Applied Microbiology, vol. 109, no. 4, pp. 1479-1486, 2010.

[21] D. K. B. Runyoro, M. I. N. Matee, O. D. Ngassapa, C. C. Joseph, and Z. H. Mbwambo, "Screening of Tanzanian medicinal plants for anti-Candida activity," BMC Complementary and Alternative Medicine, vol. 6, article 11, 2006.

[22] E. C. Sigei, M. Muturi, and C. Bii, "Antifungal activities of Camellia sinensis crude extract, mixture with milk, on selected pathogenic and mycotoxic fungi," Journal of Medicinal Plants Research, vol. 9, no. 42, pp. 1070-1080, 2015.

[23] G. S. Hall, Interactions of yeasts, moulds, and antifungal agents: how to detect resistance, Springer Science and Business Media, 2011.

[24] P. G. Mwitari, P. A. Ayeka, J. Ondicho, E. N. Matu, and C. C. Bii, "Antimicrobial activity and probable mechanisms of action of medicinal plants of kenya: withania somnifera, warbugia ugandensis, prunus africana and plectrunthus barbatus," PLoS ONE, vol. 8, no. 6, Article ID e65619, 2013.

[25] F. Opoku and O. Akoto, "Antimicrobial and phytochemical properties of Alstonia boonei extracts," Organic Chemistry: Current Research, vol. 137, no. 1, pp. 2161-0401, 2015.

[26] S. A. F. El-Nagerabi, A. E. Elshafie, S. S. AlKhanjari, S. N. AlBahry, and M. R. Elamin, "The Potential of Baobab (Adansonia digitata L.) extracts as biocontrol on the growth and aflatoxin production by Aspergillus flavus and A. parasiticus," Journal of Food Research, vol. 2, no. 3, p. 93, 2013.

[27] O. O. Shobowale, N. J. Ogbulie, E. E. Itoandon, M. O. Oresegun, and S. O. A. Olatope, "Phytochemical and antimicrobial evaluation of aqueous and organic extracts of Calotropis procera ait leaf and latex," Nigerian Food Journal, vol. 31, no. 1, pp. 77-82, 2013.

[28] M. Iqbal, T. Ghous, A. N. Khan, and K. Akhtar, "Evaluation of antimicrobial activity of extracts of fresh and spoiled Spinacia oleracea against some mammalian pathogens," African Journal of Microbiology Research, vol. 6, no. 29, pp. 5847-51, 2012.

[29] D. A. Mahmoud, N. M. Hassanein, K. A. Youssef, and A. Zeid, "Antifungal activity of different neem leaf extracts and the nimonol against some important human pathogens," Brazilian Journal of Microbiology, vol. 42, no. 3, pp. 1007-1016, 2011.

[30] L. K. Omosa, J. O. Midiwo, V. M. Masila et al., "Cytotoxicity of 91 Kenyan indigenous medicinal plants towards human CCRFCEM leukemia cells," Journal of Ethnopharmacology, vol. 179, pp. 177-196, 2016.

[31] V. Steenkamp, A. C. Fernandes, and C. E. J. Van Rensburg, "Screening of Venda medicinal plants for antifungal activity against Candida albicans," South African Journal of Botany, vol. 73, no. 2, pp. 256-258, 2007.

[32] G. F. De Oliveira, N. A. J. C. Furtado, A. A. Da Silva Filho et al., "Antimicrobial activity of Syzygium cumini (Myrtaceae) leaves extract," Brazilian Journal of Microbiology, vol. 38, no. 2, pp. 381384, 2007.
[33] C. J. Kosgei, "Phytochemical screening, cytotoxicity studies and larvicidal activity of hexane extract of Lippia kituiensis against Rhipicephalus appendiculatus," Journal of Biomedical and Pharmaceutical Research, vol. 3, no. 4, 2014.

[34] T. G. Amabye and M. Said, "In vitro antimicrobial efficacy of fractions from Demakese (Ocimum lamifolium) leaves extract from Mekelle Tigray, Ethiopia," Natural Products Chemistry and Research, 2015.

[35] B. P. Chapagain, Z. Wiesman, and L. Tsror (Lahkim), "In vitro study of the antifungal activity of saponin-rich extracts against prevalent phytopathogenic fungi," Industrial Crops and Products, vol. 26, no. 2, pp. 109-115, 2007.

[36] J.-W. Zhang, J.-M. Gao, T. Xua et al., "Antifungal activity of alkaloids from the seeds of Chimonanthus praecox," Chemistry and Biodiversity, vol. 6, no. 6, pp. 838-845, 2009.

[37] D. Miron, F. Battisti, F. K. Silva et al., "Antifungal activity and mechanism of action of monoterpenes against dermatophytes and yeasts," Brazilian Journal of Pharmacognosy, vol. 24, no. 6, pp. 660-667, 2014.

[38] S. Handali, E. Moghimipour, B. Sadaghi-Nejad, A. Ameri, Z. Ramezani, and M. E. Azemi, "In vitro screening of anticandida activity of saponins extracted from Glycyrrhiza glabra and Quillaja saponaria," Asian Journal of Pharmaceutical and Clinical Research, vol. 1, no. 2, pp. 160-162, 2013.

[39] T. ZhaoXiu, J.-R. C. Li GuangXing, K. YuMei, and Z. Z. Wen, "Toxigenicity and pathogenicity of groundnut A. flavus strain," Fujian Journal of Agricultural Sciences, vol. 15, no. 2, pp. 19-22, 2000.

[40] G. T. de Oliveira, J. M. S. Ferreira, L. H. Rosa, E. P. de Siqueira, S. Johann, and L. A. R. D. S. Lima, "In vitro antifungal activities of leaf extracts of Lippia alba (Verbenaceae) against clinically important yeast species," Revista da Sociedade Brasileira de Medicina Tropical, vol. 47, no. 2, pp. 247-250, 2014.

[41] Y. Sahan, "Effect of prunus laurocerasus L. (Cherry Laurel) leaf extracts on growth of bread spoilage fungi," Bulgarian Journal of Agricultural Science, vol. 17, no. 1, pp. 83-92, 2011.

[42] W. Mequanint, E. Makonnen, and K. Urga, "In vivo antiinflammatory activities of leaf extracts of Ocimum lamiifolium in mice model," Journal of Ethnopharmacology, vol. 134, no. 1, pp. 32-36, 2011.

[43] A. J. De Lucca, C. Carter-Wientjes, K. A. Williams, and D. Bhatnagar, "Blue light ( $470 \mathrm{~nm}$ ) effectively inhibits bacterial and fungal growth," Letters in Applied Microbiology, vol. 55, no. 6, pp. 460-466, 2012.

[44] I. V. Bol'shakova, E. L. Lozovskaia, and I. I. Sapezhinskiü, "Photosensitizing and photoprotective properties of extracts from groups of medicinal plants," Biofizika, vol. 42, pp. 926-932, 1996.

[45] M. Sunda, F. Rosillon, and K. M. Taba, "Contribution to the water disinfection study by photosensitization with plants extracts," European Journal of Water Quality, vol. 39, no. 2, pp. 199-209, 2008.

[46] M. Razzaghi-Abyaneh and M. Rai, Antifungal Metabolites from Plants, Springer, 2013. 

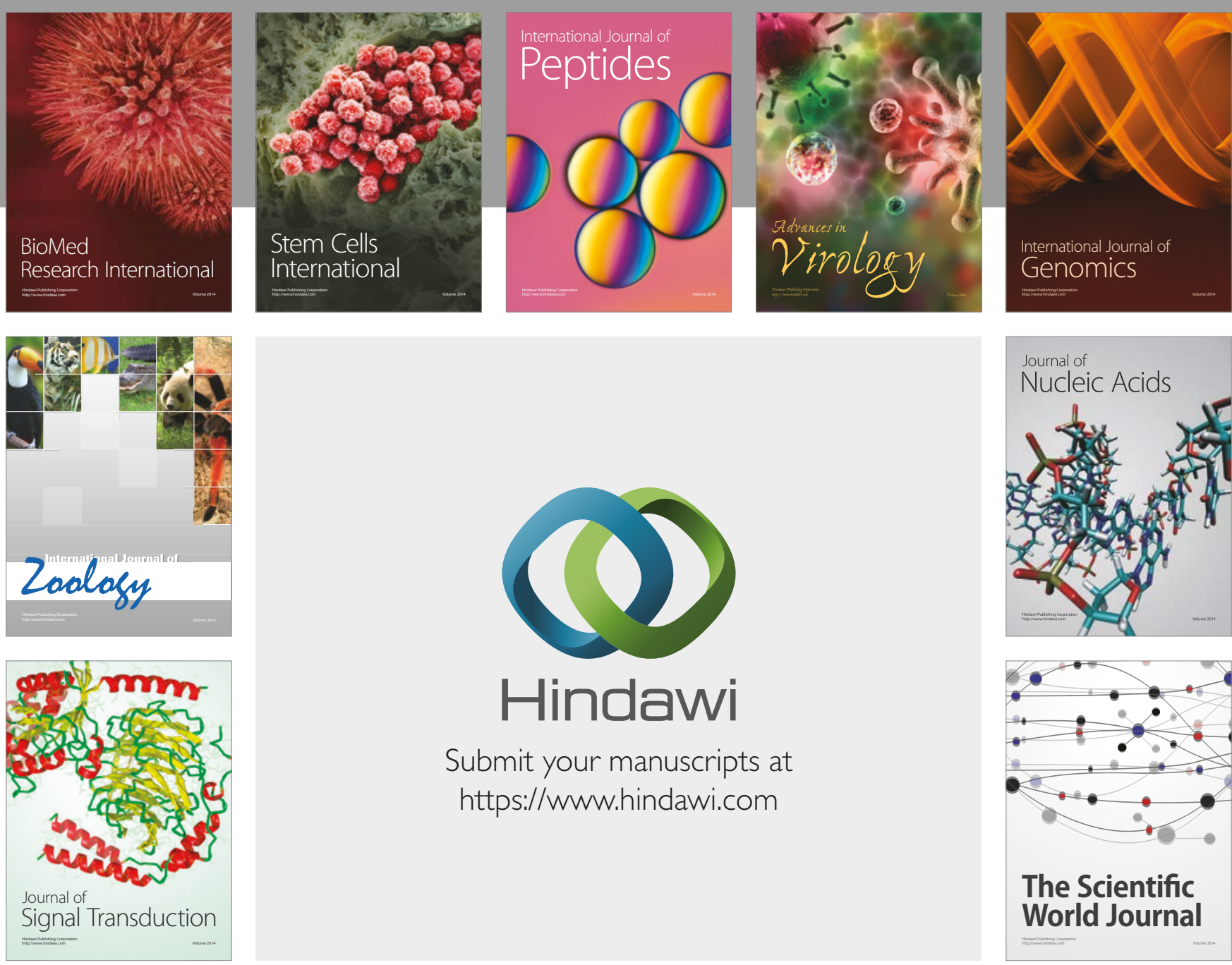

Submit your manuscripts at

https://www.hindawi.com
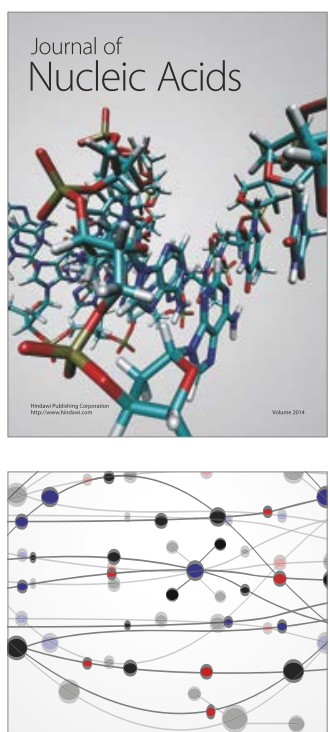

The Scientific World Journal

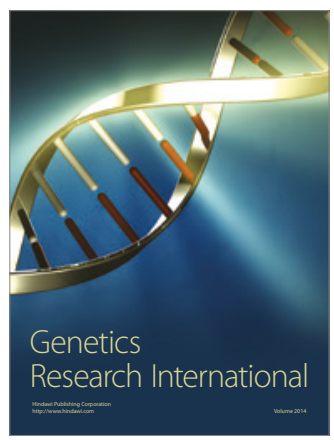

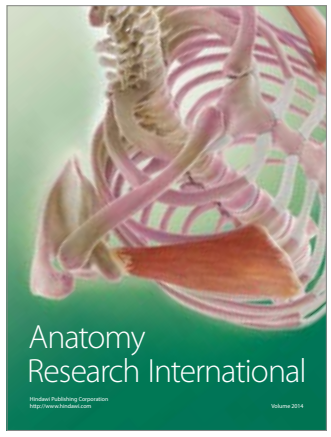

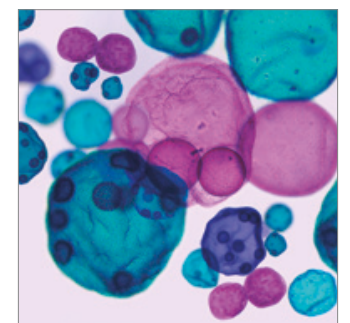

International Journal of Microbiology
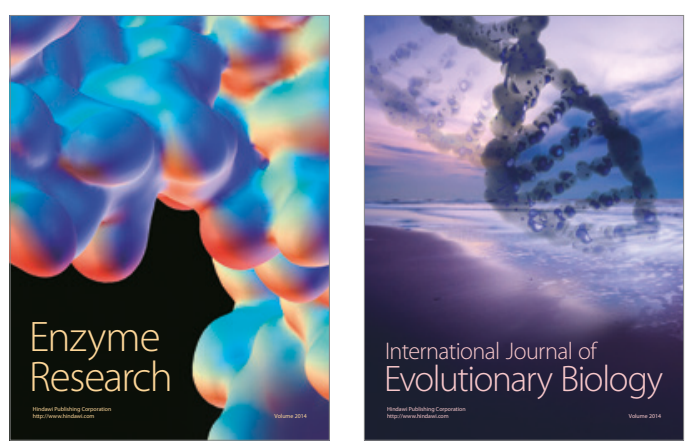
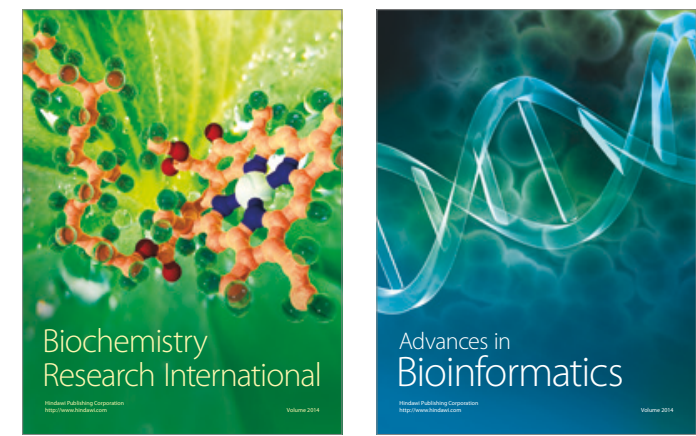

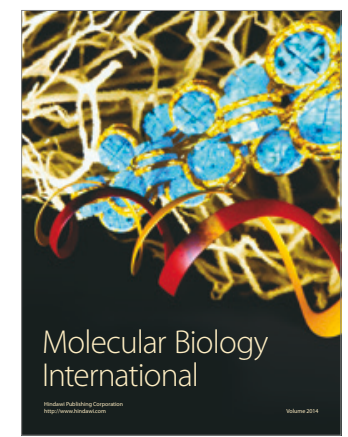

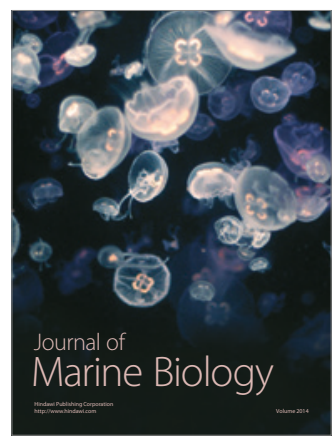

